\title{
Light mesons at BESIII
}

\author{
Xiaobin $\mathrm{Ji}^{1}$, a (for the BESIII Collaboration) \\ ${ }^{1}$ Institute of High Energy Physics, Chinese Academy of Sciences
}

\begin{abstract}
BESIII had accumulated $1.3 \times 10^{9} \mathrm{~J} / \psi$ data samples. Selected results of the light mesons study based on part or full $J / \psi$ samples are presented, including several observed structures around $1.85 \mathrm{GeV} / \mathrm{c}^{2}$, partial wave analysis of $J / \psi \rightarrow \gamma \eta \eta$, measurements of $\eta$ and $\eta^{\prime}$ anomalous decays, and $a_{0}(980)$ in $J / \psi \rightarrow p \bar{p} a_{0}(980)$.
\end{abstract}

\section{Introduction}

Hadron spectroscopy would help us understand the substructure of observed hadrons and verify the theory. Quantum Chromodynamics (QCD) describes the strong interactions of colored quarks and gluons, it predicts the existence of new types of hadrons except the ordinary meson and baryon in the quark model, such as glueballs, hybirds and multiquark states. Experimental search of new hadrons and measure the new decay modes of known hadron would provide valuable information for the further understanding the strong interactions.

The BESIII detector [1] at BEPC II (the upgraded Beijing Electron and Positron Collider), has collected the world's largest data samples at $\tau$-charm energy region since 2009, including 1.3 billion $J / \psi$ events, 0.5 billion $\psi(3686)$ events, $2.9 \mathrm{fb}^{-1}$ at the peak of the $\psi(3770)$ resonance, and lots of data samples above $4.0 \mathrm{GeV} / \mathrm{c}^{2}$, which offers us a unique opportunity to study the hadron spectroscopy and search for the new hadrons.

The article reviews several structures around 1.85 $\mathrm{GeV} / c^{2}$ found or confirmed by BESIII, the particle wave analysis results of $J / \psi \rightarrow \gamma \eta \eta$, the study of $\eta$ and $\eta^{\prime}$ anomalous decays, and the analysis of $J / \psi \rightarrow p \bar{p} a_{0}(980)$.

\section{Structures around $1.85 \mathrm{GeV} / \mathrm{c}^{2}$ in $J / \psi$ decay}

Results in this section are based on 225.3 million $J / \psi$ collected in 2009 at BESIII.

\section{$2.1 X(1860)$ and $X(1835)$}

An anomalous enhancement near the $p \bar{p}$ mass threshold in the process $J / \psi \rightarrow \gamma p \bar{p}$ was first observed by BESII experiment [2]. It was confirmed by BESIII [3] and CLEO-c experiment [4].

In order to determine the parameters of the $p \bar{p}$ mass threshold structure, a partial wave analysis (PWA) of

a. e-mail: jixb@ihep.ac.cn
$J / \psi \rightarrow \gamma p \bar{p}$ with $M_{p \bar{p}}<2.2 \mathrm{GeV} / c^{2}$ was performed [5]. In the fit, the signal amplitudes is described by the relativistic covariant tensor amplitude method [6] and the final states interaction (FSI) is included using Julich formulation [7]. The spin-parity of the $p \bar{p}$ mass threshold structure was determined to be $0^{-+}$, and the mass, width and product $\mathrm{BR}$ for the $X(p \bar{p})$ were measured to be: $M=1832_{-5}^{+19}$ (stat.) $)_{-17}^{+18}$ (syst.) \pm 19 (model) $\mathrm{MeV} / c^{2}, \Gamma=$ $13 \pm 39$ (stat.) ${ }_{-13}^{+10}$ (syst.) \pm 4 (model) $\mathrm{MeV}$ (a total width of $\Gamma<76 \mathrm{MeV} / c^{2}$ at the $90 \%$ C.L $)$ and $\mathcal{B}(J / \psi \rightarrow \gamma X) \mathcal{B}(X \rightarrow$ $p \bar{p})=\left(9.0_{-1.1}^{+0.4} \text { (stat. }\right)_{-5.0}^{+1.5}$ (syst.) $\pm 2.3($ model $\left.)\right) \times 10^{-5}$, respectively.

A PWA on $\psi^{\prime} \rightarrow \gamma p \bar{p}$ was also performed by fixing the mass, width and spin-parity of $X(p \bar{p})$ to results from $J / \psi$. The measured BRs is $\mathcal{B}\left(\psi^{\prime} \rightarrow \gamma X\right) \times \mathcal{B}(X \rightarrow$ $p \bar{p})=\left(4.57 \pm 0.36(\text { stat. })_{-4.07}^{+1.23}\right.$ (syst. $) \pm 1.28($ model $\left.)\right) \times 10^{-6}$ and the production ratio of the $X(p \bar{p})$ between $J / \psi$ and $\psi^{\prime}$ radiative decays is $R=\left(5.08_{-0.45}^{+0.71} \text { (stat. }\right)_{-3.58}^{+0.67}$ (syst.) \pm 0.12 (model))\%.

No $p \bar{p}$ threshold enhancement was found in the $J / \psi \rightarrow$ $\omega p \bar{p}[8]$, which means pure FSI is disfavored.

The $X(1835)$ was observed from the invariant mass of $\pi^{+} \pi^{-} \eta^{\prime}$ in $J / \psi \rightarrow \gamma \pi^{+} \pi^{-} \eta^{\prime}$ decay with a statistical significance of $7.7 \sigma$ by the BESII experiment [9]. The same process was studied at BESIII. X(1835) was confirmed with the statistical significance larger than $20 \sigma$ [10]. The angular distribution analysis shows that it is consistent with expectation for pseudoscalar state. Furthermore, two new structure named $X(2120)$ and $X(2370)$ were observed with the statistical significance larger than $7.2 \sigma$ and $6.4 \sigma$ respectively.

\section{$2.2 X(1870)$ in $J / \psi \rightarrow \omega \eta \pi \pi$}

To further understand the nature of $p \bar{p}$ mass threshold structure $(X(1860))$ and/or $X(1835)$, the decay of $J / \psi \rightarrow \omega \pi^{+} \pi^{-} \eta$ was studied [11]. The process of $J / \psi \rightarrow$ $\omega X(1870), X(1870) \rightarrow a_{0}^{ \pm}(980) \pi^{\mp}$ was first observed with the signal significance estimated to be $7.2 \sigma$. The $f_{1}(1285)$ and $\eta(1450)$ were also clearly observed in the $\eta \pi^{+} \pi^{-}$mass 
spectrum. Figure 1 shows results of fit to the $\eta \pi^{+} \pi^{-}$mass spectrum.

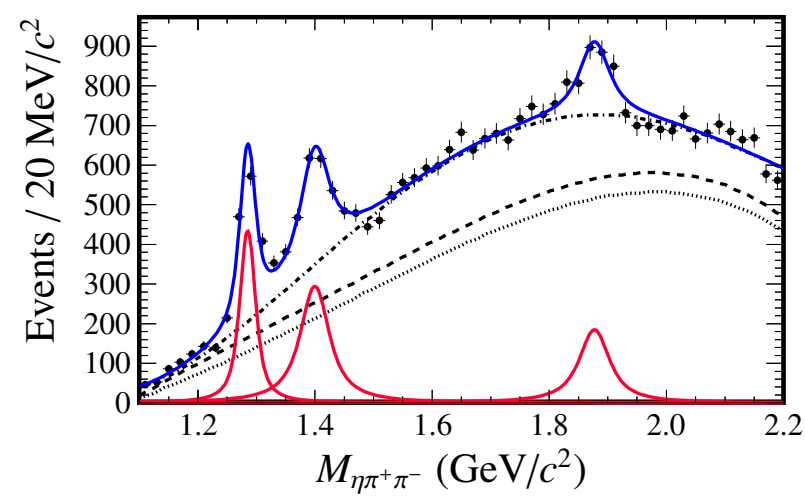

Figure 1. Results of fits to the $M\left(\eta \pi^{+} \pi^{-}\right)$mass distribution for events with either the $\eta \pi^{+}$or $\eta \pi^{-}$in the $a_{0}(980)$ mass window in $J / \psi \rightarrow \omega \eta \pi \pi$. The dotted curve shows the contribution of non- $\omega$ and/or non- $a_{0}(980)$ background, the dashed line also includes the contribution from $J / \psi \rightarrow b_{1}(1235) a_{0}(980)$, and the dot-dashed curve indicates the total background with the nonresonant $J / \psi \rightarrow \omega a_{0}^{ \pm}(980) \pi^{\mp}$ included. $\chi^{2} /$ d.o.f is 1.27 for this fit.

\section{$2.3 X(1840)$ in $J / \psi \rightarrow \gamma 3\left(\pi^{+} \pi^{-}\right)$}

Since the $X(1835)$ was confirmed to be a pseudoscalar particle [10] and it may have properties in common with the $\eta_{c}$. Six charged pions is a known decay mode of the $\eta_{c}$. Therefore, $J / \psi$ radiative decays to $3\left(\pi^{+} \pi^{-}\right)$may be a favorable channel to search for the $X$ states in the $1.8-1.9$ $\mathrm{GeV} / \mathrm{c}^{2}$ region. Fig. 2 shows the $3\left(\pi^{+} \pi^{-}\right)$invariant mass spectrum in $J / \psi \rightarrow \gamma 3\left(\pi^{+} \pi^{-}\right)$. A structure at $1.84 \mathrm{GeV} / c^{2}$ was observed with a statistical significance of $7.6 \sigma$. The mass and width were measured to be $M=1842.2 \pm 4.2_{-26}^{+7.1}$ $\mathrm{MeV} / \mathrm{c}^{2}$ and $\Gamma=83 \pm 14 \pm 11 \mathrm{MeV}$. The product branching fraction was determined to be $\mathcal{B}(J / \psi \rightarrow \gamma X(1840)) \times$ $\mathcal{B}\left(X(1840) \rightarrow 3\left(\pi^{+} \pi^{-}\right)\right)=\left(2.44 \pm 0.36_{-0.74}^{+0.60}\right) \times 10^{-5}$.

\subsection{PWA of $J / \psi \rightarrow \gamma \omega \phi$}

A study of the double OZI suppressed decays of $J / \psi \rightarrow \gamma \omega \phi$ [12] was performed. A strong deviation $(>30 \sigma)$ from three-body phase space for $J / \psi \rightarrow \gamma \omega \phi$ near the $\omega \phi$ invariant-mass threshold was observed (see Fig. 3). Assuming the enhancement is due to the influence of a resonance, the $X(1810)$, a partial wave analysis with a tensor covariant amplitude determined that the spin-parity of the $X(1810)$ is $0^{++}$. The mass and width of the $X(1810)$ were determined to be $M=1795 \pm 7(\text { stat })_{-5}^{+13}($ syst $) \pm 19(\bmod )$ $\mathrm{MeV} / c^{2}$ and $\Gamma=95 \pm 10(\text { stat })_{-34}^{+21}$ (syst) $\pm 75(\bmod ) \mathrm{MeV} / \mathrm{c}^{2}$ and the product branching fraction was measured to be $\mathcal{B}(J / \psi \rightarrow \gamma X(1810)) \times \mathcal{B}(X(1810) \rightarrow \omega \phi)=(2.00 \pm$ 0.08 (stat) $)_{-1.00}^{+0.45}$ (syst) $\left.\pm 1.30(\mathrm{mod})\right) \times 10^{-4}$, where the first error indicates the statistical error and the second is the systematical error. These results are consistent within errors with those from the BESII experiment [13].

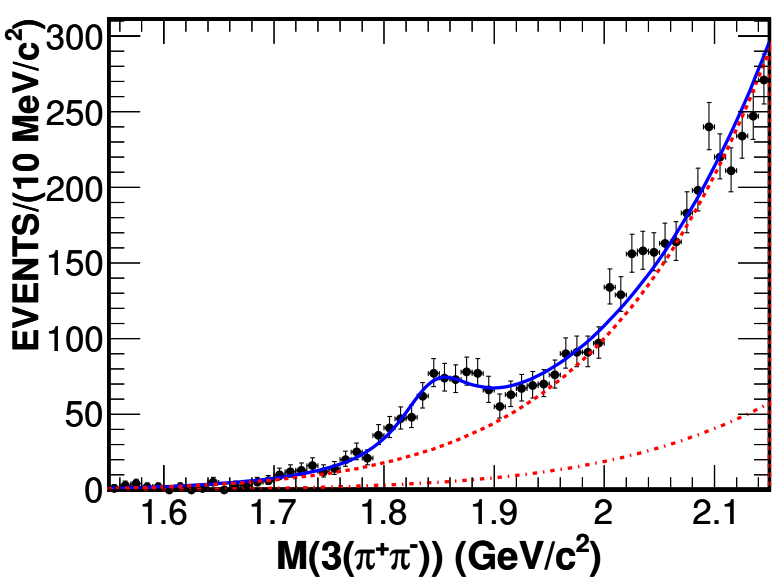

Figure 2. The fit of mass spectrum of $3\left(\pi^{+} \pi^{-}\right)$in $J / \psi \rightarrow$ $\gamma 3\left(\pi^{+} \pi^{-}\right)$. The dots with error bars are data; the solid line is the fit result. The dashed line represents all the backgrounds, including the background events from $J / \psi \rightarrow \pi^{0} 3\left(\pi^{+} \pi^{-}\right)$(dash-dotted line, fixed in the fit) and a third-order polynomial representing other backgrounds.

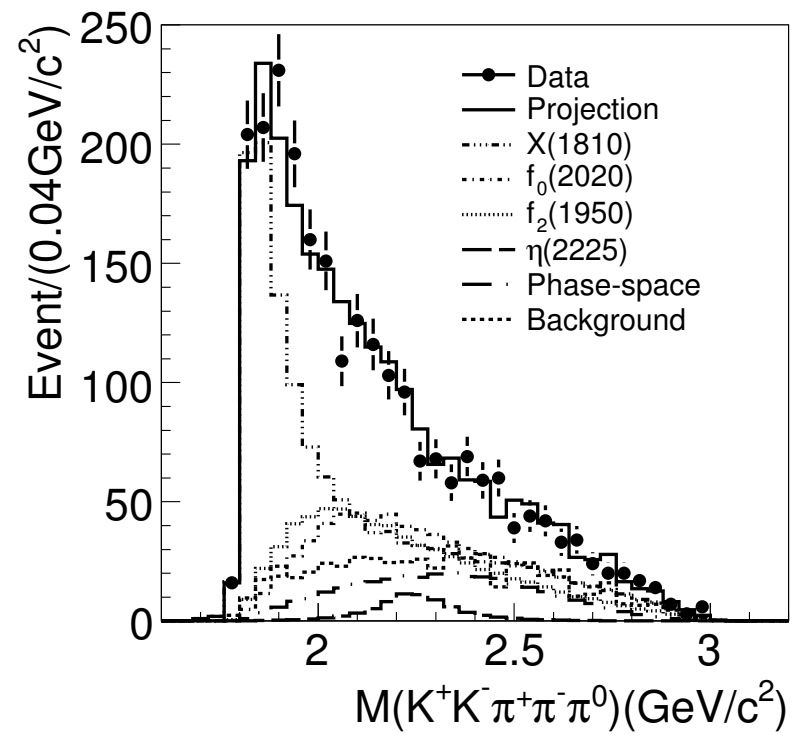

Figure 3. Comparisons between data and PWA fit projections in the invariant mass spectrum of $K^{+} K^{-} \pi^{+} \pi^{-} \pi^{0}$ in $J / \psi \rightarrow \gamma \omega \phi$.

The comparison to the mentioned BESIII results of the masses and widths are shown in Fig. 4. The mass of $X(1840)$ is in agreement with $X(p \bar{p})$, while its width is significantly broader. Therefore, based on these data, one cannot determine whether $X(1840)$ is a new state or the signal of a $3\left(\pi^{+} \pi^{-}\right)$decay mode of $X(p \bar{p})$. Further study, including an amplitude analysis to determine the spin and parity of the $X(1840)$, is needed to establish the relationship between these experimental observations. 


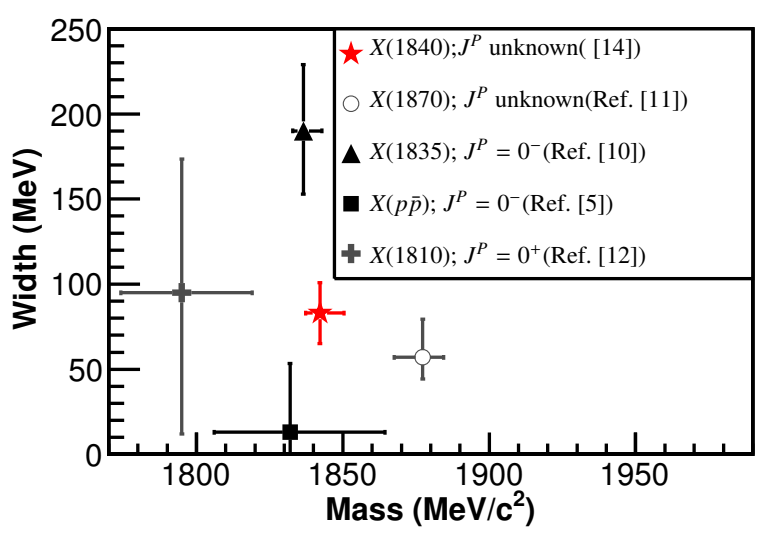

Figure 4. Comparisons of observations at BESIII. The error bars include statistical, systematic, and, where applicable, model uncertainties.

\section{PWA of $J / \psi \rightarrow \gamma \eta \eta$}

Radiative $J / \psi$ decay is a gluon-rich process and has long been regarded as one of the most promising hunting grounds for glueballs. In particular, for a $J / \psi$ radiative decay to two pseudoscalar mesons, it offers a very clean laboratory to search for scalar and tensor glueballs because only intermediate states with $J^{P C}=e^{e v e n^{++}}$are possible.

Using 225 million $J / \psi$ events collected with the BESIII detector, a PWA of $J / \psi \rightarrow \gamma \eta \eta$ has been performed. Fig. 5 shows the projection of PWA in the invariant mass spectrum of $\eta \eta$. The scalar contributions are mainly from $f_{0}(1500), f_{0}(1710)$ and $f_{0}(2100)$, while no evident contributions from $f_{0}(1370)$ and $f_{0}(1790)$ are seen. Recently, the production rate of the pure gauge scalar glueball in $J / \psi$ radiative decays predicted by the lattice QCD [15] was found to be compatible with the production rate of $J / \psi$ radiative decays to $f_{0}(1710)$; this suggests that $f_{0}(1710)$ has a larger overlap with the glueball compared to other glueball candidates (eg. $f_{0}(1500)$ ). In this analysis, the production rate of $f_{0}(1710)$ and $f_{0}(2100)$ are both about one order of magnitude larger than that of the $f_{0}(1500)$ and no clear evidence was found for $f_{0}(1370)$, which are both consistent with, at least not contrary to, lattice QCD predictions.

The tensor components, which are dominantly from $f_{2}^{\prime}(1525), f_{2}(1810)$ and $f_{2}(2340)$, also have a large contribution in $J / \psi \rightarrow \gamma \eta \eta$ decays. The significant contribution from $f_{2}^{\prime}(1525)$ is shown as a clear peak in the $\eta \eta$ mass spectrum; a tensor component exists in the mass region from $1.8 \mathrm{GeV} / c^{2}$ to $2 \mathrm{GeV} / c^{2}$, although we cannot distinguish $f_{2}(1810)$ from $f_{2}(1910)$ or $f_{2}(1950)$; and the PWA requires a strong contribution from $f_{2}(2340)$, although the possibility of $f_{2}(2300)$ cannot be ruled out.

\section{$4 \eta$ and $\eta^{\prime}$ physics}

The $\eta / \eta^{\prime}$ system provides a unique stage for understanding the distinct symmetry-breaking mechanisms. Furthermore, the $\eta / \eta^{\prime}$ decays play an important role to explore the effective theory of QCD at low energy, especially

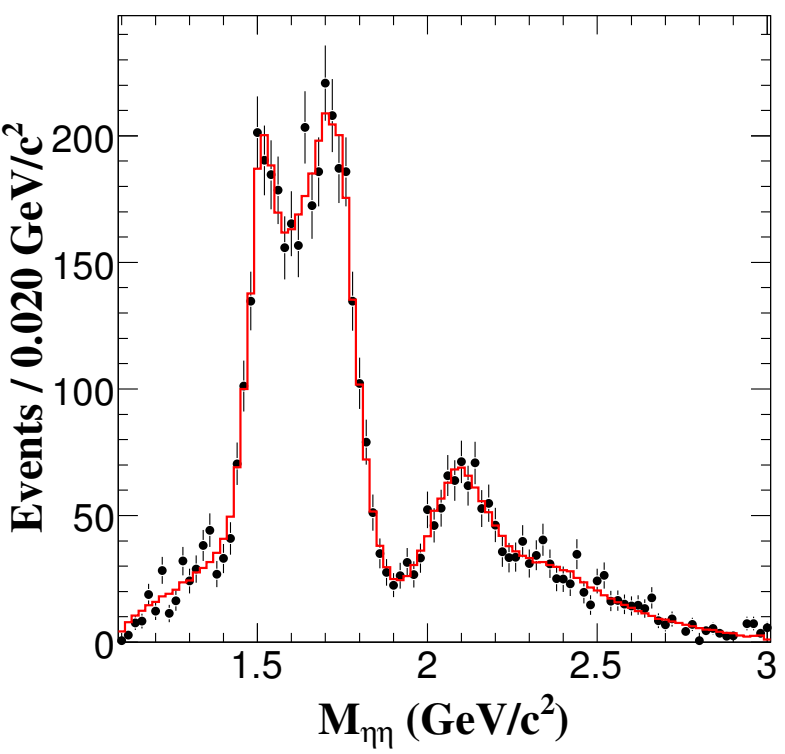

Figure 5. The invariant mass of $\eta \eta$ (dots with error bars) and the PWA fit projections (histogram).

for the Chiral Perturbation Theory ( $\chi$ PTh). Its main decay modes, including hadronic and radiative decays, have been well measured, but the study of anomalous decays is still an open field. BESIII had collected 1.3 billion $J / \psi$ events, one can obtain large $\eta / \eta^{\prime}$ samples $\left(\sim 10^{6}\right)$ from processes $J / \psi \rightarrow \gamma \eta / \eta^{\prime}$ or $J / \psi \rightarrow \phi \eta / \eta^{\prime}$. It is a good place to study the anomalous decays of $\eta / \eta^{\prime}$. BESIII had published results of $\eta / \eta^{\prime}$ invisible decays [16], weak decay of $\eta / \eta^{\prime} \rightarrow \pi^{-} e^{+} v+c . c$. [17]. Here we present other three studies briefly.

\subsection{Measurement of $\eta^{\prime} \rightarrow \pi^{+} \pi^{-} l^{+} l^{-}$}

$\eta^{\prime} \rightarrow \pi^{+} \pi^{-} e^{+} e^{-}$was first reported by CLEO recently [18]. Theoretically this decay is expected to proceed via a virtual photon intermediate state, $\eta^{\prime} \rightarrow \pi^{+} \pi^{-} \gamma^{*} \rightarrow$ $\pi^{+} \pi^{-} e^{+} e^{-}$, and provides a more stringent test of the theories since it involves off-shell photons.

Based on $225 \mathrm{M} \mathrm{J} / \psi$ events, the decays of $\eta^{\prime} \rightarrow$ $\pi^{+} \pi^{-} l^{+} l^{-}$were studied via $J / \psi \rightarrow \gamma \eta^{\prime}$ at BESIII [19]. A clear $\eta^{\prime}$ signal was observed in the $\pi^{+} \pi^{-} e^{+} e^{-}$invariant mass spectrum, and the branching fraction was measured to be $\mathcal{B}\left(\eta^{\prime} \rightarrow \pi^{+} \pi^{-} e^{+} e^{-}\right)=(2.11 \pm 0.12 \pm 0.15) \times 10^{-3}$, which is in good agreement with theoretical predictions and the previous measurement, but was determined with much higher precision. No $\eta^{\prime}$ signal was found in the $\pi^{+} \pi^{-} \mu^{+} \mu^{-}$mass spectrum, and the upper limit was determined to be $\mathcal{B}\left(\eta^{\prime} \rightarrow \pi^{+} \pi^{-} \mu^{+} \mu^{-}\right)<2.9 \times 10^{-5}$ at the $90 \%$ confidence level.

\subsection{Observation of $\eta^{\prime} \rightarrow \pi^{+} \pi^{-} \pi^{+} \pi^{-}\left(\pi^{+} \pi^{-} \pi^{0} \pi^{0}\right)$}

The strong decays $\eta^{\prime} \rightarrow \pi^{+} \pi^{-} \pi^{+(0)} \pi^{-(0)}$ are not suppressed by approximate symmetries. They are expected 
to be mediated by chiral anomalies, since an odd number (five) of pseudoscalar particles are involved. In particular, a contribution from a new type of anomaly, the pentagon anomaly, might show up. There should be also a significant contribution from the intermediate state with two $\rho$ mesons. Based on a sample of 1.3 billion $J / \psi$ events taken with the BESIII detector, we observe the decay modes $\eta^{\prime} \rightarrow \pi^{+} \pi^{-} \pi^{+} \pi^{-}$and $\eta^{\prime} \rightarrow \pi^{+} \pi^{-} \pi^{0} \pi^{0}$ with a statistical significance of $18 \sigma$ and $5 \sigma$, respectively (See Fig.6. The branching fractions were determined to be $\mathcal{B}\left(\eta^{\prime} \rightarrow \pi^{+} \pi^{-} \pi^{+} \pi^{-}\right)=(8.53 \pm 0.69 \pm 0.64) \times 10^{-5}$ and $\mathcal{B}\left(\eta^{\prime} \rightarrow \pi^{+} \pi^{-} \pi^{0} \pi^{0}\right)=(1.82 \pm 0.35 \pm 0.18) \times 10^{-4}$, which are consistent with the theoretical predictions based on a combination of chiral perturbation theory and vector-meson dominance [20], but not with the broken- $\mathrm{SU}_{6} \times \mathrm{O}_{3}$ quark model [21].
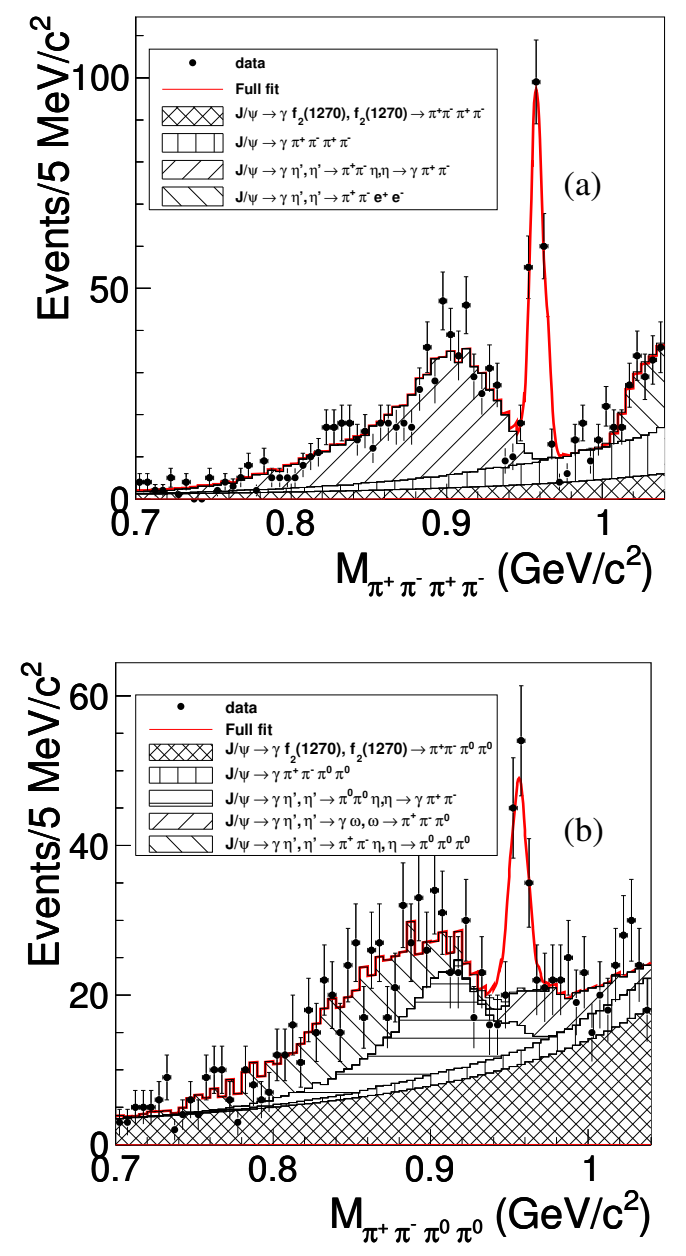

Figure 6. Results of the fits to (a) $M_{\pi^{+} \pi^{-} \pi^{+} \pi^{-}}$and (b) $M_{\pi^{+} \pi^{-} \pi^{0} \pi^{0}}$, where the background contributions are displayed as the hatched histograms.

\section{3 $\eta / \eta^{\prime} \rightarrow \gamma \gamma \pi^{0}$ (Preliminary)}

The decay $\eta \rightarrow \pi^{0} \gamma \gamma$, in the frame of $\chi \mathrm{PTh}$, both at $O\left(p^{2}\right)$ and $O\left(p^{4}\right)$ is forbidden because there is no direct coupling of photons to the neutral $\pi^{0}$ and $\eta$. The first sizable contribution comes at $O\left(p^{6}\right)$ [22]. This decay provides a unique opportunity to test directly the correctness of the calculations of third order $\chi \mathrm{PTh}$.

With a sample of 1.3 billion $J / \psi$ events in the BESIII, the rare, doubly radiative decays $\eta / \eta^{\prime} \rightarrow \gamma \gamma \pi^{0}$ have been studied. $\eta^{\prime}$ can be seen clearly in the invariant mass spectrum of $\gamma \gamma \pi^{0}$ (Fig. 7), the peaking background events mainly come from $\eta^{\prime} \rightarrow \gamma \omega(\rho)$ with $\omega(\rho) \rightarrow \gamma \pi^{0}$. The branching fraction of $\eta^{\prime} \rightarrow \gamma \gamma \pi^{0}$ is measured for the first time to be $\mathcal{B}\left(\eta^{\prime} \rightarrow \gamma \gamma \pi^{0}\right)=(6.91 \pm 0.51 \pm 0.58) \times 10^{-4}$. The measured value is much lower than that of the theoretical predictions $[23,24]$ but consistent with the upper limit in PDG. No evidence for the decay of $\eta \rightarrow \gamma \gamma \pi^{0}$ is found.

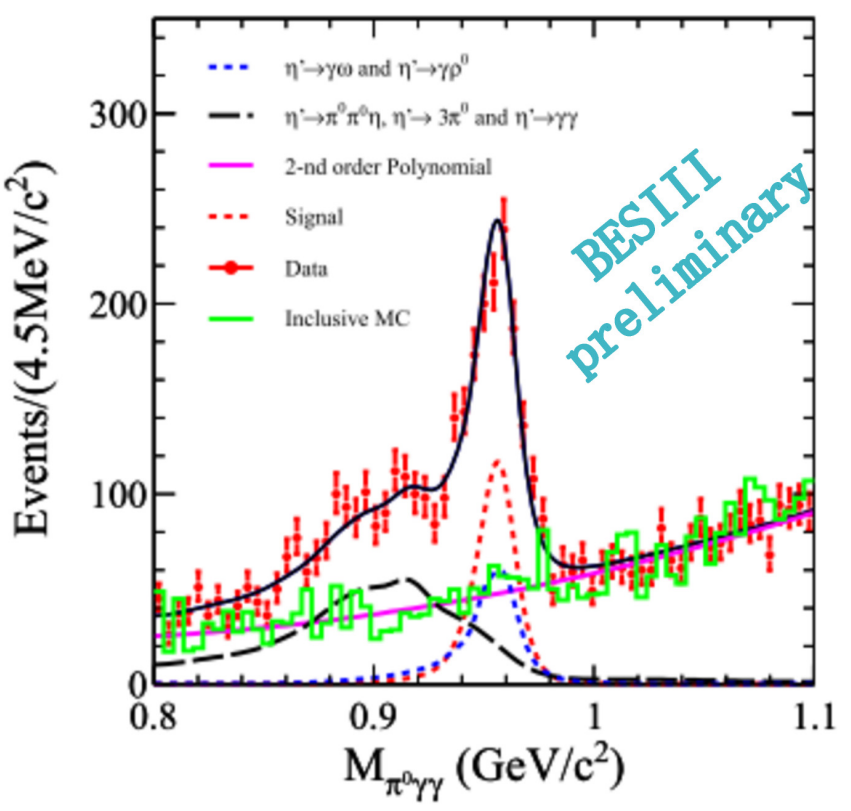

Figure 7. The $M_{\gamma \gamma \pi^{0}}$ distribution with fit results superimposed for the $\eta^{\prime} \rightarrow \gamma \gamma \pi^{0}$ decay.

\section{$\left.5 J / \psi \rightarrow p \bar{p} a_{0}(980)\right)$}

As one of the low-lying scalars, the state $a_{0}(980)$ has turned out to be mysterious in the quark model scenario. Its production near threshold allows tests of various hypotheses for its structure. The measurement of $J / \psi \rightarrow$ $p \bar{p} a_{0}(980)$ is an additional observable constraining any phenomenological models trying to understand the nature of the $a_{0}(980)$. A chiral unitary coupled channels approach of the $\chi \mathrm{PTh}$ is applied in investigation of the four-body decays $J / \psi \rightarrow N \bar{N} M M$ process [25] where the $N$ stands for a baryon and the $M$ for a meson. The experiment input is needed for further progress in understanding of the dynamics of the four-body decay processes taking the FSI of mesons into account.

Based on 225 million $J / \psi$ events at BESIII, $J / \psi \rightarrow$ $p \bar{p} a_{0}(980), a_{0}(980) \rightarrow \pi^{0} \eta$ was observed for the fist time with a statistical significance of $3.2 \sigma$ [26]. Fig. 8 shows the fit to the invariant mass spectrum of $\pi^{0} \eta$. Without considering the interference between the signal channel and the 
same final states with intermediate $N^{*}$ states, the branching fraction was measured to be $\mathcal{B}\left(J / \psi \rightarrow p \bar{p} a_{0}(980) \rightarrow\right.$ $\left.p \bar{p} \pi^{0} \eta\right)=(6.8 \pm 1.2 \pm 1.3) \times 10^{-5}$. Taking the branching fraction of $J / \psi \rightarrow p \bar{p} \pi^{+} \pi^{-}$from PDG, the coefficient $r_{4}$ in the chiral unitary approach [25] shows preference to $r_{4}=0.2$ instead of -0.27 .

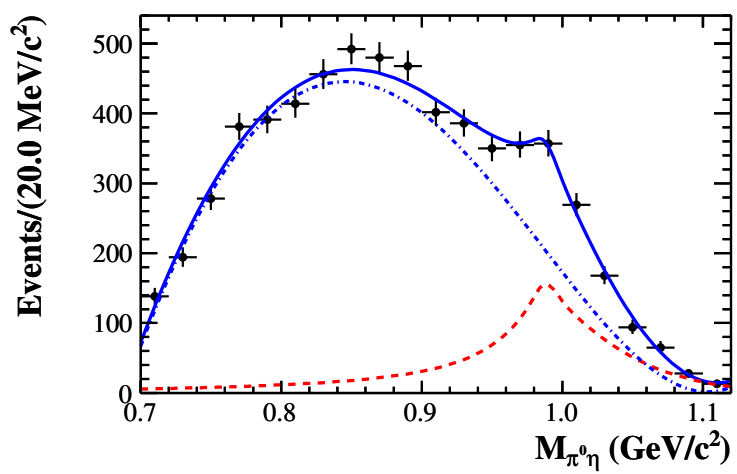

Figure 8. The results of fitting the mass spectrum for $\pi^{0} \eta$. Dots with error bars are data and the solid line is the fitted spectrum. The dash-dotted line shows the non- $a_{0}(980)$ background described by a third-order Cheybechev polynomial. The dashed line shows the signal described by an efficiency-weighted Flatté formula convoluted with a resolution function.

\section{Summary}

BESIII had collected 1.3 billion $J / \psi$ data samples. Based 225 million or full $J / \psi$ samples, some progresses on the light meson study are reported. $p \bar{p}$ threshold enhancement in $J / \psi \rightarrow \gamma p \bar{p}, X(1835)$ in $J / \psi \rightarrow \gamma \eta^{\prime} \pi^{+} \pi^{-}, X(1810)$ in $J / \psi \rightarrow \gamma \omega \phi$ were confirmed. Two new structures, $X(1840)$ in $J / \psi \rightarrow \gamma 3\left(\pi^{+} \pi^{-}\right)$and $X(1870)$ in $J / \psi \rightarrow \omega \eta \pi \pi$ were observed. Furthermore, various studies on the light meson were shown, including PWA of $J / \psi \rightarrow \gamma \eta \eta, \eta / \eta^{\prime}$ anomalous decays, $a_{0}(980)$ in $J / \psi \rightarrow p \bar{p} \eta \pi$. It turns out that BESIII is an excellent place to study the hadron spectroscopy. With the high statistics data accumulated at the BESIII, more interesting results are expected to be coming soon.

\section{References}

[1] M. Ablikim et al. (BESIII Collaboration), Nucl. Instrum. Meth. A614, 345 (2010), 0911.4960
[2] J. Bai et al. (BES Collaboration), Phys. Rev. Lett. 91, 022001 (2003), hep-ex/0303006

[3] M. Ablikim et al. (BESIII Collaboration), Chin. Phys. C34 (2010), 1001. 5328

[4] J. Alexander et al. (CLEO Collaboration), Phys. Rev. D82, 092002 (2010), 1007.2886

[5] M. Ablikim et al. (BESIII Collaboration), Phys. Rev. Lett. 108, 112003 (2012), 1112 . 0942

[6] S. Dulat, B.S. Zou, Eur. Phys. J. A26, 125 (2005), hep-ph/0508087

[7] A. Sibirtsev, J. Haidenbauer, S. Krewald, U.G. Meissner, A.W. Thomas, Phys. Rev. D71, 054010 (2005), hep-ph/0411386

[8] M. Ablikim et al. (BESIII Collaboration), Phys. Rev. D87, 112004 (2013), 1303.3108

[9] M. Ablikim et al. (BES Collaboration), Phys. Rev. Lett. 95, 262001 (2005), hep-ex/0508025

[10] M. Ablikim et al. (BESIII Collaboration), Phys. Rev. Lett. 106, 072002 (2011), 1012. 3510

[11] M. Ablikim et al. (BESIII Collaboration), Phys. Rev. Lett. 107, 182001 (2011), 1107.1806

[12] M. Ablikim et al. (BESIII Collaboration), Phys. Rev. D87, 032008 (2013), 1211. 5668

[13] M. Ablikim et al. (BES Collaboration), Phys. Rev. Lett. 96, 162002 (2006), hep-ex/0602031

[14] M. Ablikim et al. (BESIII Collaboration), Phys. Rev. D88, 091502 (2013), 1305. 5333

[15] L.C. Gui, Y. Chen, G. Li, C. Liu, Y.B. Liu et al., Phys. Rev. Lett. 110, 021601 (2013), 1206.0125

[16] M. Ablikim et al. (BESIII Collaboration), Phys. Rev. D87, 012009 (2013), 1209. 2469

[17] M. Ablikim et al. (BESIII Collaboration), Phys. Rev. D87, 032006 (2013), 1211. 3600

[18] P. Naik et al. (CLEO Collaboration), Phys. Rev. Lett. 102, 061801 (2009), 0809. 2587

[19] M. Ablikim et al. (BESIII Collaboration), Phys. Rev. D87, 092011 (2013), 1303.7360

[20] F.K. Guo, B. Kubis, A. Wirzba, Phys. Rev. D85, 014014 (2012), 1111.5949

[21] D. Parashar, Phys. Rev. D19, 268 (1979)

[22] L. Ametller, J. Bijnens, A. Bramon, F. Cornet, Phys. Lett. B276, 185 (1992)

[23] R. Escribano, PoS CD12, 035 (2013)

[24] R. Jora, Nucl. Phys. Proc. Suppl. 207-208, 224 (2010)

[25] C.b. Li, E. Oset, M. Vicente Vacas, Phys. Rev. C69, 015201 (2004), nucl-th/0305041

[26] M. Ablikim et al. (BESIII Collaboration), Phys. Rev. D90, 052009 (2014), 1408. 3938 